\title{
TCT and test beam results of irradiated magnetic Czochralski silicon (MCz-Si) detectors
}

\author{
P. Luukka ${ }^{\mathrm{a}, *}$, J. Härkönen ${ }^{\mathrm{a}}$, T. Mäenpääa ${ }^{\mathrm{a}}$, B. Betchart ${ }^{\mathrm{b}}$, S. Czellar ${ }^{\mathrm{a}}$, R. Demina ${ }^{\mathrm{b}}$, A. Furgeric ${ }^{\mathrm{c}}$, Y. Gotra ${ }^{\mathrm{b}}$, M. Frey $^{\mathrm{c}}$, F. Hartmann $^{\mathrm{c}}$, S. $^{\text {. }}$ \\ Korjenevski $^{b}$, M.J. Kortelainen ${ }^{\text {a }}$, T. Lampén ${ }^{\mathrm{a}}$, B. Ledermann ${ }^{\mathrm{c}}$, V. Lemaitre ${ }^{\text {, }}$, T. Liamsuwan ${ }^{\mathrm{c}}$, O. Militaru ${ }^{\text {d }}$, H. Moilanen ${ }^{\mathrm{a}}$, H.J.

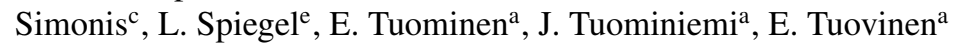 \\ ${ }^{a}$ Helsinki Institute of Physcis, Helsinki, Finland \\ ${ }^{b}$ Department of Physics and Astronomy, University of Rochester, NY, USA \\ ${ }^{c}$ Universität Karlsruhe (TH), Institut für Experimentelle Kernphysik, Karlsruhe, Germany \\ ${ }^{d}$ Université catholique de Louvain, Louvain-la-Neuve, Belgium \\ ${ }^{e}$ Fermi National Accelerator Laboratory, Batavia, IL, USA
}

\begin{abstract}
Pad and strip detectors processed on high resistivity n-type magnetic Czochralski silicon (MCz-Si) were irradiated to several different fluences with protons. The pad detectors were characterized with the Transient Current Technique (TCT) and the full-size strip detectors with a reference beam telescope and a $225 \mathrm{GeV}$ muon beam. The TCT measurements indicate a double junction structure and space charge sign inversion in MCz-Si detectors after $6 \times 10^{14} 1 \mathrm{MeV} \mathrm{n}$ eq $/ \mathrm{cm}^{2}$ fluence. In the beam test a S/N of 50 was measured for a non-irradiated $\mathrm{MCz}-\mathrm{Si}$ sensor, and a S/N of 20 for the sensors irradiated to the fluences of $1 \times 10^{14} 1 \mathrm{MeV}$ $\mathrm{n}_{\mathrm{eq}} / \mathrm{cm}^{2}$, and $5 \times 10^{14} 1 \mathrm{MeV} \mathrm{n} \mathrm{n}_{\mathrm{eq}} / \mathrm{cm}^{2}$.
\end{abstract}

Key words:

Magnetic Czochralski silicon, double junction, beam test, Transient Current Technique

PACS: 71.55.Cn, 29.40.Gx, 29.40.Wk

\section{Introduction}

There is a strong physics case for eventually increasing the LHC luminosity by an order of magnitude. The planned LHC upgrade scenario, the Super-LHC (SLHC) entails a factor of ten increase in the integrated luminosity, which would correspond to expected total fluences of fast hadrons above $1 \times 10^{16}$ $\mathrm{cm}^{-2}$. This is well beyond the radiation tolerance of any standard p-on-n Float Zone silicon (Fz-Si) devices used in the current large scale experiments such as CMS and ATLAS Trackers. Furthermore, with the higher luminosity, also the detector occupancy will increase correspondingly. This means that the new SLHC tracker systems should be more radiation hard and provide higher resolution than the current tracker systems to address the increased occupancy.

Oxygen enrichment of silicon bulk has widely been acknowledged to improve the radiation tolerance of silicon detectors $[1,2,3]$. For example ATLAS experiment decided to use Diffusion Oxygenated Float Zone silicon (DOFz-Si) in their pixel detectors that will receive the highest fluence during the 10 years of LHC operation. However, in DOFz-Si the oxygen is introduced into the silicon material by high-temperature longterm diffusion, which increases the risk of contaminating the silicon wafers. However, Czochralski silicon $(\mathrm{Cz}-\mathrm{Si})$ contains intrinsically a high concentration of oxygen $\left(>5 \times 10^{17} \mathrm{~cm}^{-3}\right)$ and developments in the $\mathrm{Cz}-\mathrm{Si}$ crystal growth technology have

${ }^{*}$ Corresponding author. Address: CERN, CH-1211 Geneva 23, Tel: +4122 767 8783, E-mail:panja.luukka@cern.ch enabled the production of $\mathrm{Cz}$-Si wafers, for example by magnetic Czochralski (MCz) method, with sufficiently high resistivity and with well-controlled, high concentration of oxygen. Furthermore, since $\mathrm{Cz}-\mathrm{Si}$ is commonly used semiconductor material in the microelectronics industry, it is available in large quantities and many commercial foundries are familiar with its processing. These aspects are important when constructing large tracking systems, which can consist of hundreds of square meters of silicon sensors.

The MCz-Si material has been studied extensively in the RD50 and RD39 collaborations [4, 5] and it has been found to be more radiation hard against protons than traditional $\mathrm{Fz}-\mathrm{Si}$ or DOFz-Si in terms of the depletion voltage behavior. In large scale systems the power consumption of the tracking system is an important factor, and thus a lower full depletion voltage can be an asset, when choosing the material for the SLHC detector applications. However, most of the radiation hardness studies have been done on pad detectors. Yet, in order to prove that the MCz-Si material is suitable for any large-scale tracking system like the SLHC CMS tracker, it is necessary to perform extensive tests on full-size segmented devices in addition to pad detector characterization. For example, the charge collection efficiency (CCE) is partly determined by the electric field distribution in silicon. In irradiated silicon, the electric field distribution is complicated and contains for example a so-called Double Junction (DJ) structure [6]. Moreover, the electric field distribution is very different in a pad detector compared to a segmented device. This affects the detector weighting field, i.e. the electrostatic coupling between the drifting charge and the 
sensing electrode. In a simple diode the weighting field is inversely proportional to the thickness of the device and hence the induced charge that has been generated by a traversing particle is proportional to the length of the drift. However, in a segmented device due to the different electric field shape, the moving charge induces most charge in the electrode, when it is close to it and not through the entire device thickness [7]. Furthermore, the space charge sign inversion (SCSI) of the detector bulk affects the CCE. Despite extensive research, the SCSI phenomenon is poorly understood in the potentially radiation hard silicon materials such as $\mathrm{MCz}-\mathrm{Si}$. However, it is assumed that in a segmented detector the SCSI will manifest itself as a degraded cluster resolution after the type inversion fluence.

In this experiment we tested the performance of several proton irradiated silicon sensors processed on magnetic Czochralski silicon wafers. The pad detectors were characterized with Transient Current Technique (TCT) and the full-size strip detectors with a reference telescope in a $225 \mathrm{GeV}$ muon beam.

\section{Detector processing and irradiation}

The MCz-Si detectors used in this study were processed with a simple six mask level process at the Helsinki University of Technology Centre for Micro and Nanotechnology (Micronova) facility. The starting material was 4 inch n-type wafers with a thickness of $300 \pm 2 \mu \mathrm{m}$ and $\langle 100\rangle$ crystal orientation grown with the $\mathrm{MCz}$ method by Okmetic Ltd., Finland. A detailed process description can be found from the reference [8].

The large area strip detectors had 768 channels and an area of $4.1 \times 4.1 \mathrm{~cm}^{2}$. In the detector design the strip pitch was 50 $\mu \mathrm{m}$, strip width $10 \mu \mathrm{m}$ and the strip length $3.9 \mathrm{~cm}$. The pad detectors had $5 \times 5 \mathrm{~mm}^{2} \mathrm{p}^{+}$implanted area and a $2 \times 2 \mathrm{~mm}^{2}$ opening in the front metallization for the TCT measurements.

Two full-size strip sensors were irradiated with $26 \mathrm{MeV}$ protons (Universität Karlsruhe) to fluences of $1 \times 10^{14} 1 \mathrm{MeV}$ $\mathrm{n}_{\mathrm{eq}} / \mathrm{cm}^{2}$, and $5 \times 10^{14} 1 \mathrm{MeV} \mathrm{n} \mathrm{n}_{\mathrm{eq}} / \mathrm{cm}^{2}$, and one was used as a non-irradiated reference. The pad detectors were irradiated with $24 \mathrm{GeV}$ protons (CERN PS) to several different neutron equivalent fluences. The devices were not annealed prior to their characterization with the beam telescope or the TCT setup.

\section{Transient Current Technique measurements}

In short, the TCT measurement is based on the detection of the dominant type of charge carrier, electron or hole, which drifts across the whole detector thickness after being excited by a photon. Detailed description of this method can be found from the references [9] and [10]. It is important to note that when the electric field of an irradiated detector is analyzed, the measured signal is affected by the charge trapping into the radiation-induced defects. The influence of the trapping can be deducted from the measured data by applying values obtained from literature $[11,12,13]$.

In this experiment the pad detectors were characterized with the RD39 Collaboration Cryogenic TCT-setup, which is capable of operating below liquid nitrogen temperature. The TCT setup consists of a high bandwidth oscilloscope, a Keithley source meter unit capable of sourcing up to $500 \mathrm{~V}$, a vacuum chamber, a cold finger, a Leybold helium stirling cooler, temperature and vacuum control units, a LabVIEW-based data acquisition system and two different lasers emitting at $670 \mathrm{~nm}$ and $1060 \mathrm{~nm}$. A detailed description of the setup can be found from reference [14].

\section{Beam test measurements}

The detector measurements in the beam were done with a reference telescope and $225 \mathrm{GeV}$ muon beam at the CERN $\mathrm{H} 2$ test beam. The telescope is based on the CMS detector readout prototype components and HPK strip sensors with a $60 \mu \mathrm{m}$ readout pitch and intermediate strips in between. The impact point precision of the reference tracks at the location of the detector under test is about $4 \mu \mathrm{m}$, the telescope has a $\mathrm{S} / \mathrm{N}$ of 28 and an active area of $4 \times 4 \mathrm{~cm}^{2}$. The telescope and the detectors under test are housed inside a cold chamber, in which the operating temperature can be set by two water cooled $350 \mathrm{~W}$ Peltier elements to the desired value. Detailed description of the beam test setup can be found from reference [15].

Detailed description of the off-line calibration and data analysis can be found from reference [16]. In a few words, first the pedestal and noise levels for each strip are determined from dedicated calibration runs without a beam. Then, the cluster candidates are constructed from physics runs around strips with a high signal to noise ratio $(\mathrm{S} / \mathrm{N})$, and the hit position is computed with the centre of gravity method. A three-dimensional track is reconstructed, if a compatible hit is found on all reference planes providing measurements in the alternate coordinates perpendicular to the beam line. The Hit and Impact Points (HIP) method [17] is used to align the individual detectors in two translational and one rotational degree of freedom. Finally, with the reconstructed tracks, the hit resolution and efficiency of the test detector can be calculated.

\section{Results and discussion}

Figure 1 presents the trapping corrected ( $3 \mathrm{~ns}$ ) TCT measurement data from a pad detector irradiated to a neutron equivalent fluence of $6 \times 10^{14} 1 \mathrm{MeV} \mathrm{n}$ eq $/ \mathrm{cm}^{2}$. In the figure the current transient indicates a double junction structure i.e. electric field existing on both $\mathrm{p}^{+}$and $\mathrm{n}^{+}$sides of the detector. This can be seen in the behavior of the transient curve with increasing voltage. First with $200 \mathrm{~V}$ the first peak dominates, but already with $300 \mathrm{~V}$ the second peak is larger than the first one, and the effect is even more visible in the curves taken with higher voltages. On the other hand, with voltages higher than the depletion voltage, the current transient is clearly increasing, and thus, the transient also indicates that the space charge sign inversion has occured. The temperature of the measurements was approximately $-30^{\circ} \mathrm{C}$, and the measurements were done with the 670 $\mathrm{nm}$ red laser.

With the beam telescope a $\mathrm{S} / \mathrm{N}$ of 50 was measured for the non-irradiated sensor and a $\mathrm{S} / \mathrm{N}$ of 20 for both irradiated sensors 
at the full depletion. Figure 2 presents the measured signal in ADC counts and Figure 3 the measured noise.

As can be seen from the Figure 2, the detector with lower fluence $\left(1 \times 10^{14} 1 \mathrm{MeV} \mathrm{n}\right.$ eq $\left./ \mathrm{cm}^{2}\right)$ depletes at around $150 \mathrm{~V}$ and the detector with higher fluence $\left(5 \times 10^{14} 1 \mathrm{MeV} \mathrm{n}\right.$ eq $\left./ \mathrm{cm}^{2}\right)$ at approximately $300 \mathrm{~V}$. Both are lower than the full depletion voltage of the non-irradiated device, which is approximately 330 V. These full depletion voltages correlate well with our former measurements that indicate a full depletion voltage minimum at approximately $2 \times 10^{14} 1 \mathrm{MeV} \mathrm{n}$ eq $/ \mathrm{cm}^{2}$ fluence for the $\mathrm{MCz}$ $\mathrm{Si}$ material irradiated with protons. Interestingly, it looks like the amount of measured signal at the full depletion is approximately $70 \%$ of the signal of the non-irradiated device for both of the irradiated devices. The only difference seems to be in the voltage with which the highest charge collection is obtained. Moreover, the device with the lower fluence has higher noise than the device irradiated to $5 \times 10^{14} 1 \mathrm{MeV} \mathrm{n}$ eq $/ \mathrm{cm}^{2}$ fluence. This is most probably due to a difference in the measurement temperature, since the device with the lower fluence was measured in $0^{\circ} \mathrm{C}$ and the device with higher fluence in $-15^{\circ} \mathrm{C}$ temperature.

Figure 4 indicates that the resolutions of the detectors are only slightly affected by the irradiation. The resolution of the non-irradiated detector is $11 \mu \mathrm{m}$, whereas the detector with the lower fluence has a resolution of $12 \mu \mathrm{m}$ and the detector with the higher fluence a resolution of $13 \mu \mathrm{m}$ at the full depletion. Thus, after the fluence of $5 \times 10^{14} 1 \mathrm{MeV} \mathrm{n}$ eq $/ \mathrm{cm}^{2}$ the $\mathrm{MCz}$ Si detector resolution has deteriorated only $2 \mu \mathrm{m}$, and is still better than the calculated digital resolution of $14 \mu \mathrm{m}(\mathrm{p} / \sqrt{12}$, where $\mathrm{p}$ is the pitch) of the device. Thus, it can be concluded that the type inversion of the material affects the resolution only marginally. This is most probably due to the double junction effect, which is also evident in the TCT-measurement data presented in Figure 1.

\section{Conclusions}

The TCT measurement results show that after the neutron equivalent fluence of $6 \times 10^{14} 1 \mathrm{MeV} \mathrm{n}$ eq $/ \mathrm{cm}^{2}$ the MCz-Si material has gone through type inversion. In addition, the data indicates a double junction structure. In the beam test a $\mathrm{S} / \mathrm{N}$ of 50 was measured for a non-irradiated $\mathrm{MCz}-\mathrm{Si}$ sensor, and a $\mathrm{S} / \mathrm{N}$ of 20 for the sensors irradiated with $1 \times 10^{14} 1 \mathrm{MeV} \mathrm{n}$ eq $/ \mathrm{cm}^{2}$ and $5 \times 10^{14} 1 \mathrm{MeV} \mathrm{n}$ eq $/ \mathrm{cm}^{2}$ fluences. The detector resolutions were $11 \mu \mathrm{m}, 12 \mu \mathrm{m}$ and $13 \mu \mathrm{m}$, respectively. Thus, the SCSI affects the resolution only marginally. This is most probably due to the double junction effect.

\section{Acknowledgements}

We wish to express our thanks Dr. Dragoslav-Laza Lazic and the CMS HCAL collaboration for their valuable technical assistance and co-operation at the CERN H2 experimental area. This work has partially been funded by the Academy of Finland. The results have been obtained in the frameworks of CERN CMS, RD50 and RD39 collaborations.

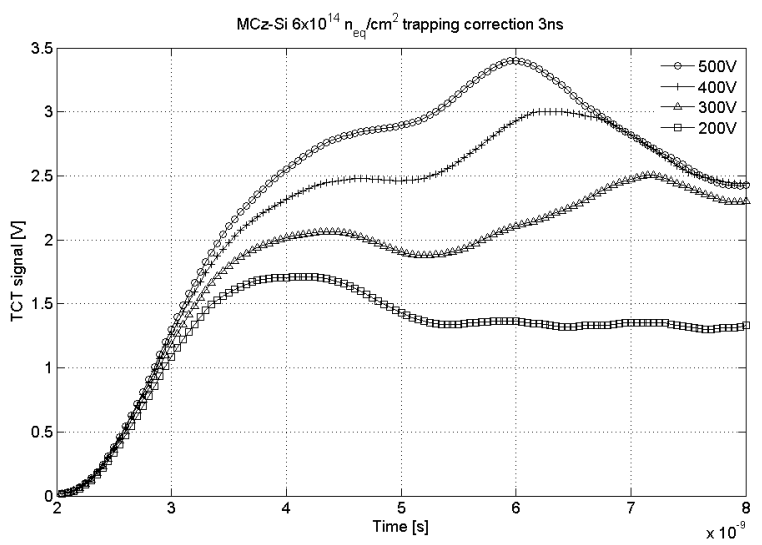

Figure 1: Trapping corrected (3ns) TCT measurement data from a sample irradiated with $24 \mathrm{GeV}$ protons to the neutron equivalent fluence of $6 \times 10^{14} 1$ $\mathrm{MeV} \mathrm{n} \mathrm{n}_{\mathrm{eq}} / \mathrm{cm}^{2}$.

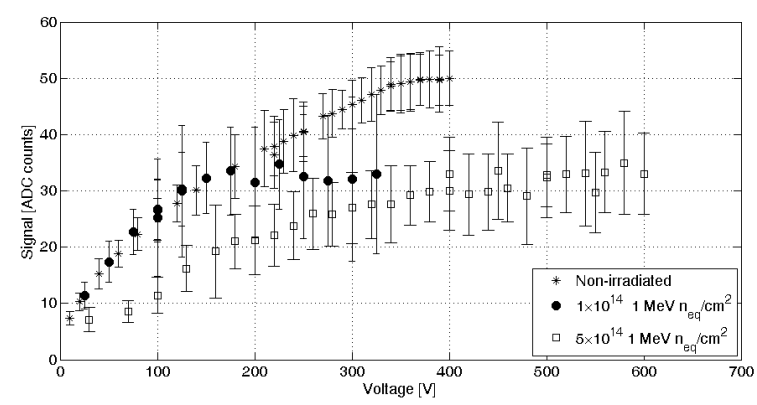

Figure 2: $\mathrm{S}$ values measured with $225 \mathrm{GeV}$ muon beam from a non-irradiated detector and detectors irradiated to $1 \times 10^{14} 1 \mathrm{MeV} \mathrm{n}$ eq $/ \mathrm{cm}^{2}$ and $5 \times 10^{14} 1$ $\mathrm{MeV} \mathrm{n}_{\mathrm{eq}} / \mathrm{cm}^{2}$ fluences. 


\section{References}

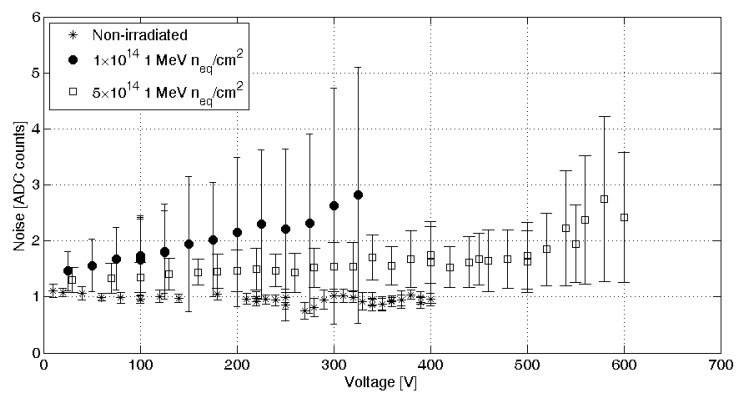

[1] Z. Li et al., IEEE Trans. Nucl. Sci. 39 (1992) 1730.

[2] Z. Li et al., IEEE Trans. Nucl. Sci. 42 (1995) 387.

[3] G. Lindstroem et el., Nucl. Instr. and Meth. A 466 (2001) 308.

[4] RD50 Status Report 2007, CERN-LHCC-2008-001.

[5] RD39 Collaboration Status Report 2007, CERN-LHCC-2007-028.

[6] E. Verbitskaya et al., Nucl. Instr. and Meth. A 583 (2007) 77.

[7] G. Kramberger, D. Contarato, Nucl. Instr. and Meth. A560 (2006) 98.

[8] J. Hrknen, et al., Nucl. Instr. and Meth. A 514 (2003) 173.

[9] V. Eremin, Nucl. Instr. and Meth. A 372 (1996) 388.

[10] V. Eremin et al., Nucl. Instr. and Meth. A 372 (1996) 188.

[11] Alison G.Bates and Michael Moll, Nucl. Instr. and Meth. A 555 (2005) 113.

[12] O. Krasel, Ph.D. thesis, Dortmund University (2004).

[13] G. Kramberger et al., Nucl. Instr. and Meth. A 481 (2002) 297.

[14] J. Hrknen et al., Nucl. Instr. and Meth. A 581 (2007) 347.

[15] T. Menp et al., Nucl. Instr. and Meth. A 593 (2008) 523.

[16] M.J. Kortelainen et al., Offline-Calibration and Data Analysis for the Silicon Beam Telescope on the CERN H2 Beam, submitted to Nucl. Instr. and Meth. A.

[17] V. Karimki et al., arXiv:physics/0306034.

Figure 3: $\mathrm{N}$ values measured with $225 \mathrm{GeV}$ muon beam from a non-irradiated detector and detectors irradiated to $1 \times 10^{14} 1 \mathrm{MeV} \mathrm{n}$ eq $/ \mathrm{cm}^{2}$ and $5 \times 10^{14} 1$ $\mathrm{MeV} \mathrm{n} \mathrm{ne}_{\mathrm{eq}} / \mathrm{cm}^{2}$ fluences.

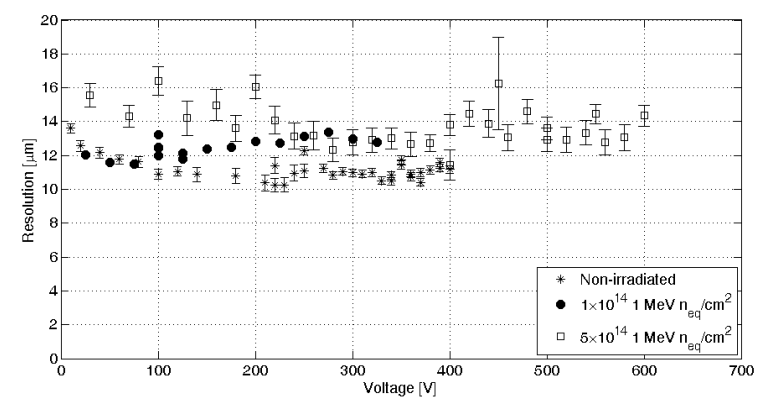

Figure 4: Resolution of the non-irradiated detector and detectors irradiated to $1 \times 10^{14} 1 \mathrm{MeV} \mathrm{n}_{\mathrm{eq}} / \mathrm{cm}^{2}$ and $5 \times 10^{14} 1 \mathrm{MeV} \mathrm{n} \mathrm{n}_{\mathrm{eq}} / \mathrm{cm}^{2}$ fluences measured with $225 \mathrm{GeV}$ muon beam. 